\title{
Clinical variables serve as prognostic factors in a model for survival from glioblastoma multiforme: an observational study of a cohort of consecutive non-selected patients from a single institution
}

Signe Regner Michaelsen ${ }^{1}$, lb Jarle Christensen², Kirsten Grunnet ${ }^{1}$, Marie-Thérése Stockhausen ${ }^{1}$, Helle Broholm³, Michael Kosteljanetz ${ }^{4}$ and Hans Skovgaard Poulsen ${ }^{1^{*}}$

\begin{abstract}
Background: Although implementation of temozolomide (TMZ) as a part of primary therapy for glioblastoma multiforme (GBM) has resulted in improved patient survival, the disease is still incurable. Previous studies have correlated various parameters to survival, although no single parameter has yet been identified. More studies and new approaches to identify the best and worst performing patients are therefore in great demand.

Methods: This study examined 225 consecutive, non-selected GBM patients with performance status (PS) 0-2 receiving postoperative radiotherapy with concomitant and adjuvant TMZ as primary therapy. At relapse, patients with PS $0-2$ were mostly treated by reoperation and/or combination with bevacizumab/irinotecan (BEV/IRI), while a few received TMZ therapy if the recurrence-free period was $>6$ months.

Results: Median overall survival and time to progression were 14.3 and 8.0 months, respectively. Second-line therapy indicated that reoperation and/or BEV/RI increased patient survival compared with untreated patients and that BEV/IRI was more effective than reoperation alone. Patient age, ECOG PS, and use of corticosteroid therapy were significantly correlated with patient survival and disease progression on univariate analysis, whereas p53, epidermal growth factor receptor, and $\mathrm{O}^{6}$-methylguanine-DNA methyltransferase expression (all detected by immunohistochemistry), tumor size or multifocality, and extent of primary operation were not. A model based on age, ECOG PS, and corticosteroids use was able to predict survival probability for an individual patient.
\end{abstract}

Conclusion: The survival of RT/TMZ-treated GBM patients can be predicted based on patient age, ECOG PS, and corticosteroid therapy status.

Keywords: Bevacizumab, Glioblastoma multiforme, Prognosis, Temozolomide

\section{Background}

Glioblastoma multiforme (GBM) is the most common adult primary brain tumor [1] and patients generally have a dismal prognosis with a median survival of just 15 months [2]. Newly diagnosed patients often undergo surgical tumor resection and studies have shown that the extent of surgical resection is correlated with increased median survival duration $[3,4]$. Given that surgery as a single

\footnotetext{
* Correspondence: hans.skovgaard.poulsen@regionh.dk

'Department of Radiation Biology, The Finsen Center, Copenhagen University Hospital, Blegdamsvej 9, DK-2100 Copenhagen, Denmark

Full list of author information is available at the end of the article
}

treatment is insufficient due to a diffuse infiltration by tumor tissue into the brain, patients generally receive concomitant and adjuvant chemotherapy with temozolomide (TMZ) in combination with radiotherapy (RT) [2,5]. TMZ is an alkylating agent that induces cell death primarily through the formation of $\mathrm{O}^{6}$-methylguanine DNA adducts, resulting in DNA double-strand breaks [6]. The drug is well tolerated with mostly mild to moderate adverse events [7]. Preclinical studies have shown that TMZ sensitizes GBM cells to RT $[8,9]$, which might explain why the combination is favorable. However, despite the EORTC-NCIC trial originally showing a survival benefit in all patients 
treated with RT/TMZ, 5-year follow-up analysis showed that nearly all patients experienced relapse and only $9.8 \%$ survived 5 years after initial diagnosis [10].

The response to and survival following RT/TMZ therapy has been correlated with several patient-specific variables. The most frequently reported predictive variables include patient age, performance status (PS), extent of surgical resection, and expression of $\mathrm{O}^{6}$-methylguanine-DNA methyltransferase (MGMT) [11-16], a DNA repair protein inhibiting the effect of TMZ by reversing alkylation [17]. Predictive variables that have been less frequently reported include tumor size [12], corticosteroid therapy [14], and positivity for a number of overexpressed or mutated molecules, including epidermal growth factor receptor (EGFR), and $\mathrm{p} 53[18,19]$.

Although GBM tends to recur locally [20], repeat surgery is only a treatment option for a limited number of patients due to poor PS, large tumor volumes, and involvement of critical brain areas [21]. As an alternative, patients with relapsed tumors have received chemotherapy or different kinds of molecular-targeted therapies [5]. Among the latter is bevacizumab (BEV), a humanized monoclonal antibody targeting vascular endothelial growth factor (VEGF). VEGF promotes proliferation, survival, and migration of endothelial cells, and is expressed and released mainly from tumor cells in response to pro-angiogenic stimuli [22]. GBM is one of the most vascularized tumors [23] and GBM tumors express high levels of angiogenic factors including VEGF [24]. Various studies, both retrospective and prospective, have shown that BEV with or without cytotoxic chemotherapy results in a substantive response rate and improved 6-month progression-free survival in GBM patients who have relapsed after previous RT/TMZ treatment [25]. However, the effect of BEV on overall survival (OS) has been somewhat modest, with most studies reporting median OS values of $<10$ months after initiation of BEV therapy [25].

To maximize patient survival and avoid unnecessary treatments, prognostic parameters must be taken into account when deciding which treatment modality is most appropriate for the individual patient. Recursive partitioning analysis (RPA) is a tool developed in the early 1990 s with which it is possible to categorize brain cancer patients into subgroups with different median survival according to a number of clinical and therapeutic parameters [26]. GBM-specific adaptations have been developed $[27,28]$ and research has shown prognostic significance of the classification for GBM patients receiving RT with or without TMZ [27]. However, the RPA classification is somewhat crude as a prognostic tool for therapeutic decision making and is more useful for the stratification of patients in clinical trials. As an alternative, more precise prognostic calculators have been developed for GBM patients receiving RT/TMZ [14,29]. However, as the number of studies of prognostic calculators in GBM patients is limited, the approach needs further investigation.

In this study we analyzed clinical and molecular data retrospectively in a cohort of 225 newly diagnosed consecutive GBM patients treated with RT/TMZ as primary therapy. Parameters identified to correlate to tumor progression and patient survival were assembled in a prognostic model able to predict patient survival. In addition, the effects of repeat surgery, BEV plus irinotecan (IRI) therapy, and the combination of both therapeutic modalities were compared for the treatment of relapsed tumors.

\section{Methods}

This study was performed according to the Declaration of Helsinki and Danish legislation. Permissions were given from the Danish Data Protection Agency (2006-41-6979) and the ethical committee for the Capital Region of Denmark (H-C-2008-095).

\section{Patients}

This study included a consecutive series of 225 patients with newly diagnosed GBM (WHO grade IV) recruited from 2005 to 2010 who were not selected other than having ECOG PS 0-2. There were 80 women and 145 men with a median age of 59.2 years (range, 22.6-75.4 years). Of these, 198 patients presented with a single tumor, while 26 patients had multifocal disease (data missing, $n=1$ ). ECOG PS was $0(n=132), 1(n=66)$, or $2(n=19)$ [data missing, $n=8]$. Patient demographics are shown in Table 1.

\section{Treatments}

Patients underwent surgery, taking either a tumor biopsy $(n=29)$ or resulting in partial $(n=104)$ or complete tumor resection $(n=89)$ prior to additional therapy (data missing, $n=3$ ). The extent of surgical radicality was based on the impression of the surgeon.

Patients received 6 weeks of concomitant RT/TMZ therapy as primary treatment. They received TMZ $75 \mathrm{mg} /$ $\mathrm{m}^{2} /$ day plus RT at a dose of 60 Gy to the planning target volume in 30 fractions with 5 fractions/week delivered by a megavoltage linear accelerator. Cerebral CT was performed with 3-mm slices and fused with baseline MRI for treatment planning. Treatment planning was performed three-dimensionally using Eclipse $^{\mathrm{Tm}}$ treatment planning system (Varian Medical Systems, Palo Alto, CA) and volumes of interest were defined in agreement with International Commission on Radiation Units \& Measurements Reports 50 and 62. The gross tumor volume (GTV) was defined as the contrast-enhanced tumor on postcontrast T1 image and/or the non-enhancing area on the T2 image on the baseline MRI scan. The clinical target volume (CTV) as defined as the GTV $+2 \mathrm{~cm}$ margin, except for bony structures. Meningeal structures were considered anatomic barriers to tumor spread, if appropriate 
Table 1 Patient demographics, therapy, and response ( $N=\mathbf{2 2 5})$

Age (years), median (range)
Gender, $n$ (\%)
Female
Male
ECOG performance status, $n$ (\%)
0
1
2
Missing
Multifocal Disease, $n(\%)$
Yes
No
Missing

Extent of tumor resection, $n(\%)$

Biopsy

Partial resection

Gross total resection

Missing

Corticosteroid therapy at initiation of RT/TMZ, n (\%)

Yes

No

Missing

No. of TMZ cycles following initial RT/TMZ, n (\%)

Median

$\begin{array}{lc}0 & 36(16.0) \\ 1 & 13(5.8) \\ 2 & 54(24.0) \\ 3 & 12(5.3) \\ 4 & 10(4.4) \\ 5 & 23(10.2) \\ 6 & 75(33.3) \\ \text { Missing } & 2(0.9)\end{array}$

Reoperation, $n$ (\%)

Yes

No

Second-line TMZ therapy, $n$ (\%)

Yes

No

Second-line BEV/IRI therapy

Yes

No

Missing

Follow-up duration (months), median (range)
$59.2(22.6-75.4)$

80 (35.6)

$145(64.4)$

$132(58.7)$

66 (29.3)

$19(8.4)$

8 (3.6)

26 (11.6)

198 (88)

1 (0.4)

29 (12.9)

104 (46.2)

89 (39.6)

$3(1.3)$

165 (73.3)

57 (25.3)

$3(1.3)$

3

36 (16.0)

3 (5.8)

54 (24.0)

$12(5.3)$

0 (4.4)

3 (10.2)

$2(0.9)$

$74(33.0)$

151 (67.0)

$12(5.3)$

$213(94.7)$

85 (37.8)

132(58.7)

8 (3.6)

60 (23-92)
Table 1 Patient demographics, therapy, and response ( $N=\mathbf{2 2 5})$ (Continued)

\begin{tabular}{lc}
\hline Best clinical response, $n(\%)$ & $6(2.7)$ \\
CR & $17(7.5)$ \\
PR & $93(41.3)$ \\
SD & $94(41.8)$ \\
PD & $15(6.7)$ \\
Missing & \\
\hline $\begin{array}{l}\text { Abbreviations: } C R \text { complete response, } P D \text { progressive disease, } P R \text { partial } \\
\text { response, } R T \text { radiotherapy, SD stable disease, TMZ temozolomide. }\end{array}$
\end{tabular}

clinically. If present, the surgical cavity was included. The internal target volume was identical to the CTV. No variations in size, shape or position of CTV in relation to anatomical reference structures were considered. Planning target volume was defined as the CTV $+0.5 \mathrm{~cm}$ margin for patient setup inconsistencies. Tolerance doses for organs at risk were as described by Emami et al. [30].

During this treatment, patients were also given antibiotic prophylaxis with $400 \mathrm{mg}$ sulfamethoxazole/80 mg trimethoprim 3 times/week. In addition, a number of patients received corticosteroid therapy to relieve neurological symptoms: 165 patients (73\%) received corticosteroid therapy at the initiation of RT/TMZ therapy.

Four weeks after completion of initial therapy, patients were given up to six courses of adjuvant TMZ therapy, with one course defined as TMZ for 5 days followed by 23 days without therapy. The initial course was given at a dose of $150 \mathrm{mg} / \mathrm{m}^{2} /$ day and the remaining courses at a dose of $200 \mathrm{mg} / \mathrm{m}^{2} /$ day. The dose was adjusted based on relevant blood tests. The number of adjuvant TMZ therapy courses given is summarized in Table 1 .

As therapy for recurrent tumors, patients who maintained ECOG PS 0-2 were initially considered for secondary surgery to remove as much tumor as possible. These patients were thereafter considered for secondary therapy with TMZ $150-200 \mathrm{mg} / \mathrm{m}^{2} /$ day if they had already received 6 courses of adjuvant TMZ and thereafter had a recurrence-free period $\geq 6$ months. The courses consisted of 5 days TMZ therapy followed by 23 days without therapy. From 2006, regardless of adjuvant TMZ therapy and extent of recurrence-free period, the patients were additionally considered for second-line therapy with BEV $10 \mathrm{mg} / \mathrm{kg}$ every 2 weeks and irinotecan (IRI), as previously described [31]. In total, 74 patients underwent secondary surgery, 12 received second-line therapy with TMZ, and 85 received second-line therapy with BEV/IRI. Characterization of the therapy is detailed in Table 1.

\section{Clinical evaluation}

At treatment initiation, a full medical history was determined and patients were examined for baseline physical and neurological status. In addition, ECOG PS [32] was 
determined, routine laboratory tests (including blood chemistry and urinalysis) were performed, and MRI scans were undertaken to evaluate tumor size and location.

The median duration of observation from the day patients first received therapy to the project cut-off day (22 October 2012) was 60 months (range, 23-92 months). In this period contrast and non-contrast MRI scans were repeated after 2, 5, and 6 courses of adjuvant TMZ. Patients' neurological and clinical performance, together with corticosteroid treatment, was recorded at these time points. All patients were thereafter followed every 3 months until death or study cut-off date using the same procedures. Safety was determined using NCI-CTCAE, version 3.0, criteria [33].

\section{Histological and immunohistochemical evaluation}

Evaluations were made on formalin-fixed, paraffinembedded tissue. Tumor tissue was classified and graded as GBM according to WHO 2007 guidelines. Diagnosis was based on conventional histological and immunohistochemical (IHC) procedures, including staining with hematoxylin and eosin, glial fibrillary acidic protein (GFAP), p53, EGFR, and MGMT. For IHC, sections were pre-treated in a microwave oven with a Tris/ethylene glycol tetra-acetic acid buffer ( $\mathrm{pH} 9.0$ ) and immunostained on a DAKO Cytomation autostainer using murine monoclonal antihuman antibodies against GFAP (Z 0334, 1:6400), p53 (M 7001, 1:800), EGFR (M 7239, 1:200) [all from DAKO, Glostrup, Denmark] and MGMT (MAB16200, 1:200, Millipore, USA). The p53, EGFR, and MGMT IHC reactions were semiquantitatively evaluated according to the number of cells stained: $<10 \%, 10-25 \%, 26-50 \%$, and $>50 \%$. Staining examples are shown in Figure 1. For statistical analysis, expression evaluated as $<10 \%$ was considered negative, while $\geq 10 \%$ was considered positive.

\section{Study endpoints}

Study endpoints were time to progression (TTP), OS, OS from recurrence, response at 3 and 6 months, and best response. TTP was defined as the time from the start of RT/TMZ treatment to radiological or clinical progression. OS was defined as the time from start of RT/TMZ treatment until death from any cause, while OS from recurrence was defined as the time from tumor relapse until death from any cause.

Response was evaluated 3 and 6 months after completion of RT. Response evaluation was based on MacDonald criteria [34], considering MRI measurements of contrastenhancing tumor size and recording of the largest crosssectional area of the tumor, patient neurological status, and corticosteroid dose. Complete response (CR) was defined as complete disappearance of measurable disease by MRI, partial response (PR) as $>50 \%$ reduction of MRI contrast enhancing tumor, and progressive disease (PD)

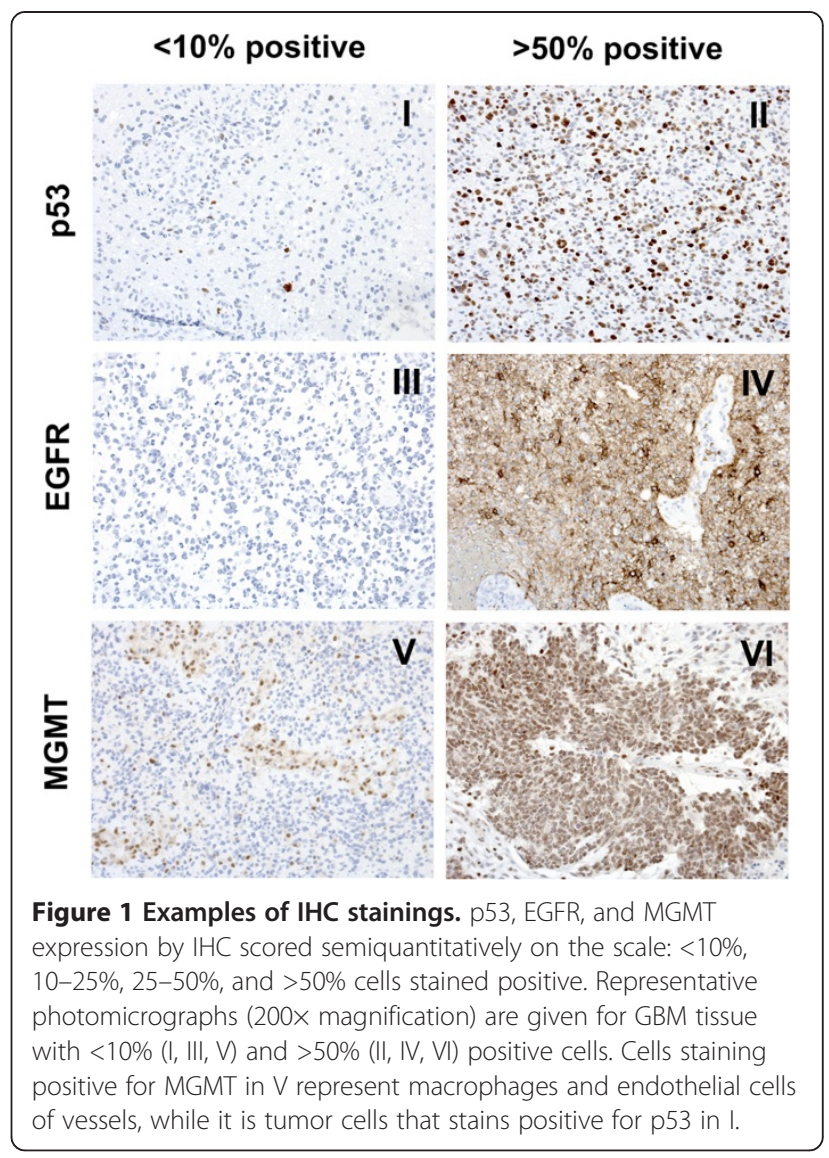

as $>25 \%$ increase in area of contrast enhancement. Patients with CR or PR also had to be taking the same or decreased corticosteroid dose and have stable or improved neurological findings. Patients, by definition, had stable disease (SD) if the criteria for CR, PR, or PD were not met and no clinical progression was observed.

For each patient, responses after 3 and 6 months were compared and a 'best response' determined, defined as the maximum achieved response registered for the patient in the observation period.

\section{Statistical considerations}

Factors that were analyzed as potential markers of prognostic significance included: age, gender, ECOG PS, extent of resection, tumor location, tumor size, previous corticosteroid therapy, and tumor EGFR, p53, and MGMT expression. Univariate and multivariate analyses of response data were performed using logistic regression analysis modeling the probability of MacDonald response at 3 and 6 months as well as the best response. Estimates of survival probabilities for OS (primary endpoint) and TTP (secondary endpoint) were performed by the Kaplan-Meier method. Univariate and multivariate analyses of OS and TTP for the chosen explanatory variables were performed using the Cox proportional hazards regression model. Analysis of time-dependent 
variables was performed using the landmark method as well as the time-dependent Cox regression model.

The final model was chosen using a backwards selection procedure, the entry level was $5 \%$. The analysis was repeated removing the least significant covariate in order to use all available data, in particular the molecular markers were only done for a subset of patients. Model assessment was done using Schoenfeld and martingale residuals. The overall concordance index (C-index) was used as a measure of discrimination $[35,36]$ and calculated in accordance to previously published guidelines [37]. In addition, a 5 fold cross validation was done to evaluate the model.

$P$ values $<.05$ were considered significant. Calculations have been performed using IBM SPSS Statistics (v19.0, IBM Denmark, Kgs. Lyngby, Denmark) and SAS (v9.2, SAS Institute, Cary, NC) software.

\section{Results}

\section{Significant factors affecting outcome from first-line} $\mathrm{RT} / \mathrm{TMZ}$

As shown in Table 1, best responses to first-line RT/TMZ among evaluable patients were: CR $(n=6 ; 2.9 \%)$; PR ( $n=17 ; 8.1 \%)$; SD $(n=93 ; 44.3 \%)$; and PD $(n=94 ; 44.8 \%)$. Data were missing for 15 patients, who were therefore not evaluated. The effects of clinical and molecular variables on best response and response at 3 and 6 months on univariate analysis are summarized in Tables 2 and 3, respectively. The only clinical variable with a significant effect on response was patient age, for which a 10-year increase resulted in a reduction of the best response $(P=.045)$. None of the other clinical factors examined had a statistically significant impact on patient best response or response at 3 and 6 months. EGFR, p53, and MGMT expression were examined as potential molecular markers for response (Table 3). Because of missing data, analyses were only available for subsets of patients: 145 of 199 patients presented EGFRpositive tumors; 105 of 202 patients presented p53positive tumors; and 65 of 163 patients presented MGMT-positive tumors. There was no significant correlation between EGFR and MGMT expression and best response or response at 3 and 6 months. The odds ratio for response at 3 months among patients with p53-positive tumors was significantly $(P=.043)$ higher as compared to those with p53-negative tumors. Although not significant, this tendency was also seen for the best response $(P=.053)$ but not for response at 6 months $(P=.21)$.

All 225 patients had TTP data, of whom 199 had disease progression. Median TTP was 8.0 months (95\% CI, 6.7-9.0 months) with progression free survival of $61 \%$ (95\% CI, 54-67\%) at 6 months and 28\% (95\% CI, 22-34\%) at 12 months (Figure 2). Increased patient age $(P=.034)$, higher ECOG PS score $(P=.046)$, and use of corticosteroid therapy at RT/TMZ initiation $(P=.036)$ had a significant

Table 2 Univariate analysis of correlation of clinical variables with survival, disease progression, and response

\begin{tabular}{|c|c|c|c|c|c|c|}
\hline Covariate & $\begin{array}{c}\text { OS } \\
(\mathrm{HR})[95 \% \mathrm{Cl}]\end{array}$ & $\begin{array}{c}\text { TTP } \\
(\mathrm{HR})[95 \% \mathrm{Cl}]\end{array}$ & $\begin{array}{l}\text { OS from } \\
\text { recurrence } \\
\text { (HR) }[95 \% \mathrm{Cl}]\end{array}$ & $\begin{array}{c}\text { Response } \\
\text { at } 3 \text { months } \\
\text { (OR) }[95 \% \mathrm{Cl}]\end{array}$ & $\begin{array}{c}\text { Response } \\
\text { at } 6 \text { months } \\
\text { (OR) }[95 \% \mathrm{Cl}]\end{array}$ & $\begin{array}{c}\text { Best } \\
\text { response } \\
(\mathrm{OR})[95 \% \mathrm{Cl}]\end{array}$ \\
\hline \multicolumn{7}{|l|}{ Operation } \\
\hline Gross total vs. biopsy & $0.76(0.49-1.17)$ & $0.74(0.48-1.16)$ & $0.81(0.51-1.27)$ & $4.33(0.54-35)$ & $1.88(0.21-16.9)$ & $4.00(0.49-32)$ \\
\hline \multirow[t]{2}{*}{ Partial vs. biopsy } & $0.98(0.64-1.50)$ & $0.86(0.56-1.32)$ & $1.05(0.68-1.65)$ & $1.37(0.15-12.2)$ & $0.86(0.09-8.22)$ & $2.13(0.25-17)$ \\
\hline & $P=.22$ & $P=.37$ & $P=.23$ & $P=.07$ & $P=.35$ & $P=.24$ \\
\hline \multirow[t]{2}{*}{ Age (per 10-year increase) } & $1.36(1.17-1.58)$ & $1.17(1.01-1.36)$ & $1.36(1.16-1.60)$ & $0.66(0.43-1.01)$ & $0.76(0.46-1.23)$ & $0.66(0.44-0.99)$ \\
\hline & $P<.0001$ & $P=.034$ & $P=.0001$ & $P=.056$ & $P=.26$ & $P=.045$ \\
\hline \multirow[t]{2}{*}{ Gender (female vs. male) } & $1.11(0.83-1.47)$ & $1.07(0.8-1.44)$ & $1.01(0.75-1.37)$ & $1.48(0.59-3.75)$ & $1.31(0.49-3.53)$ & $1.68(0.70-4.02)$ \\
\hline & $P=.47$ & $P=.64$ & $P=.94$ & $P=.41$ & $P=.29$ & $P=.24$ \\
\hline \multirow[t]{2}{*}{ Multifocal vs. single lesion } & $1.23(0.80-1.88)$ & $1.26(0.82-1.93)$ & $1.16(0.74-1.81)$ & NA & NA & NA \\
\hline & $P=.34$ & $P=.29$ & $P=.52$ & & & \\
\hline \multirow[t]{2}{*}{ Tumor size (2-fold increase) } & $1.00(0.88-1.14)$ & $0.98(0.87-1.11)$ & $1.03(0.90-1.19)$ & $1.41(0.87-2.29)$ & $1.56(0.95-2.57)$ & $1.39(0.89-2.16)$ \\
\hline & $P=.97$ & $P=.74$ & $P=.64$ & $P=.16$ & $P=.08$ & $P=.15$ \\
\hline \multirow[t]{2}{*}{ Corticosteroid therapy (yes vs. no) } & $2.13(1.49-2.86)$ & $1.41(1.02-1.92)$ & $2.17(1.54-3.03)$ & $0.44(0.17-1.11)$ & $0.78(0.28-2.17)$ & $0.57(0.23-1.39)$ \\
\hline & $P<.0001$ & $P=.036$ & $P<.0001$ & $P=.08$ & $P=.63$ & $P=.22$ \\
\hline \multicolumn{7}{|l|}{ ECOG performance status } \\
\hline 1 vs. 0 & $1.42(1.04-1.94)$ & $1.33(0.97-1.84)$ & $1.58(1.13-2.20)$ & $0.26(0.06-1.16)$ & $0.22(0.05-0.97)$ & $0.22(0.05-0.97)$ \\
\hline \multirow[t]{2}{*}{2 vs. 0} & $2.31(1.40-3.82)$ & $1.70(1.03-2.80)$ & $2.34(1.39-3.93)$ & $0.88(0.18-4.19)$ & $0.71(0.15-3.32)$ & $0.71(0.15-3.35)$ \\
\hline & $P=.0015$ & $P=.046$ & $P=.0007$ & $P=.21$ & $P=.13$ & $P=.13$ \\
\hline
\end{tabular}


Table 3 Univariate analysis of correlation of molecular markers with survival, disease progression, and response

\begin{tabular}{|c|c|c|c|c|c|c|}
\hline Covariate & $\begin{array}{c}\text { OS } \\
(\mathrm{HR})[95 \% \mathrm{Cl}]\end{array}$ & $\begin{array}{c}\text { TTP } \\
\text { (HR) }[95 \% \mathrm{Cl}]\end{array}$ & $\begin{array}{c}\text { OS from } \\
\text { recurrence } \\
(\mathrm{HR})[95 \% \mathrm{Cl}]\end{array}$ & $\begin{array}{c}\text { Response } \\
\text { at } 3 \text { months } \\
\text { (OR) }[95 \% \mathrm{Cl}]\end{array}$ & $\begin{array}{c}\text { Response } \\
\text { at } 6 \text { months } \\
\text { (OR) }[95 \% \mathrm{Cl}]\end{array}$ & $\begin{array}{c}\text { Best } \\
\text { response } \\
(\mathrm{OR})[95 \% \mathrm{Cl}]\end{array}$ \\
\hline \multicolumn{7}{|l|}{ EGFR } \\
\hline \multirow[t]{2}{*}{ Positive $(n=145)$} & $1.05(0.77-1.43)$ & $0.82(0.61-1.12)$ & $1.02(0.75-1.41)$ & $0.64(0.22-1.86)$ & $0.64(0.46-4.15)$ & $1.06(0.22-1.91)$ \\
\hline & $P=.75$ & $P=.21$ & $P=.89$ & $P=.41$ & $P=.96$ & $P=.43$ \\
\hline \multicolumn{7}{|l|}{ Negative $(n=54)$} \\
\hline \multicolumn{7}{|l|}{ Missing $(n=26)$} \\
\hline \multicolumn{7}{|l|}{ p53 } \\
\hline \multirow[t]{2}{*}{ Positive $(n=105)$} & $0.76(0.55-1.05)$ & $0.92(0.66-1.27)$ & $0.73(0.52-1.03)$ & $3.01(1.04-8.7)$ & $2.04(0.68-6.1)$ & $2.64(0.99-7.1)$ \\
\hline & $P=0.10$ & $P=.60$ & $P=.071$ & $P=.043$ & $P=.21$ & $P=.053$ \\
\hline \multicolumn{7}{|l|}{ Negative $(n=97)$} \\
\hline \multicolumn{7}{|l|}{ Missing $(n=23)$} \\
\hline \multicolumn{7}{|l|}{ MGMT } \\
\hline \multirow[t]{2}{*}{ Positive $(n=65)$} & $0.97(0.64-1.48)$ & $0.90(0.59-1.36)$ & $1.42(0.91-2.19)$ & $1.36(0.35-5.34)$ & $1.02(0.25-4.18)$ & $1.78(0.52-6.13)$ \\
\hline & $P=0.89$ & $P=0.61$ & $P=.12$ & $P=0.66$ & $P=.98$ & $P=.36$ \\
\hline \multicolumn{7}{|l|}{ Negative $(n=98)$} \\
\hline Missing $(n=62)$ & & & & & & \\
\hline
\end{tabular}

Positive and negative expression are defined by $\geq 10 \%$ and $<10 \%$ of cells, respectively, stained on immunohistochemical analysis. Abbreviations: $\mathrm{Cl}$ confidence interval, $H R$ hazard ratio, $O R$ odds ratio, $O S$ overall survival, $T T P$ time to disease progression.

negative impact on TTP (Table 2). None of the other examined clinical or molecular variables had a significant impact on TTP (Tables 2 and 3).

All 225 patients had OS data, of whom 204 (90.7\%) died during the observation period. Median OS was 14.3 months (95\% CI, 13.0-15.8 months) with an OS rate of $27.1 \%$ (95\% CI, $21-33 \%)$ at 2 years and $13.9 \%$ (95\% CI, 9.5-19.0\%) at 3 years (Figure 2). Median OS from tumor recurrence was 5.9 months (95\% CI, 5.0-6.9 months). Increased patient age $(P<.0001)$, higher ECOG PS score $(P=.0015)$, and use of corticosteroid therapy at RT/TMZ initiation $(P<.0001)$ had a significant negative impact on
OS (Table 2). Increased patient age $(P=.0001)$, higher ECOG PS score $(P=.0007)$, and use of corticosteroid therapy at RT/TMZ initiation $(P<.0001)$ also showed a significant negative correlation with decreased OS from disease recurrence. None of the other clinical covariates were significantly correlated with OS or OS from disease recurrence. None of the molecular markers (EGFR, p53, and MGMT) were significantly correlated with patient survival (Table 3 ). There was a non-significant trend for longer OS $(P=.10)$ and OS from disease recurrence $(P=.071)$ among patients with $\mathrm{p} 53$-positive tumors as compared to those with p53-negative tumors.

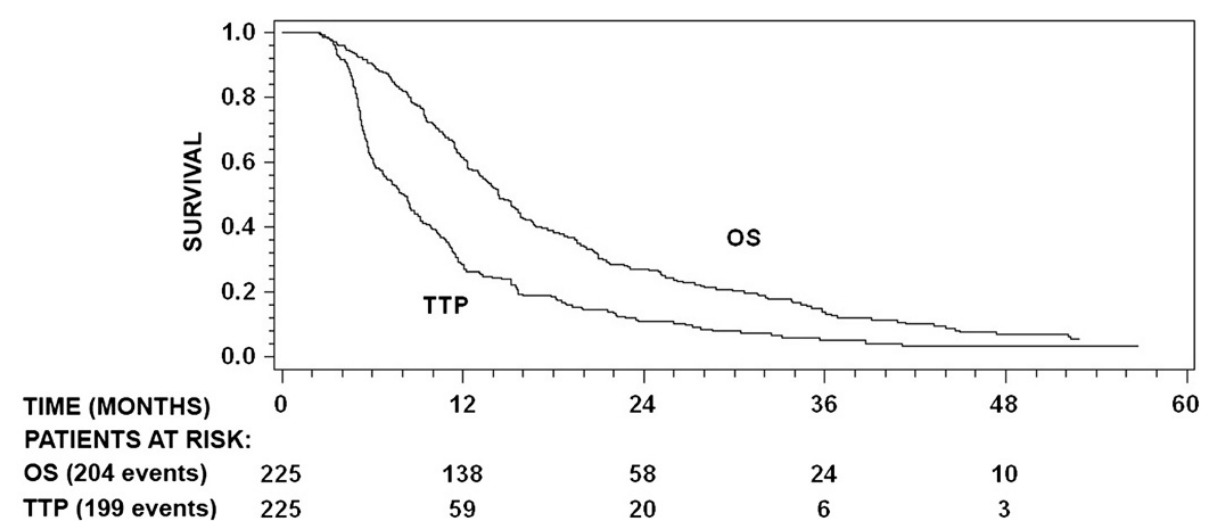

Figure 2 Kaplan-Meier plots showing TTP and OS for the patient population. The curves are based on data from all 225 examined patients. Numbers for patients at risk at selected times are shown in addition to the total number of events (deaths for OS and progression for TTP). 


\section{Reoperation and second-line BEV/IRI therapy for relapsed-tumors improve survival}

A total of 199 patients presented relapse. Most of these patients underwent reoperation of the tumor $(n=31$; $15.6 \%)$, received BEV/IRI therapy $(n=42 ; 21.1 \%)$, or had a combination of both modalities $(n=43 ; 21.6 \%)$ for recurrent disease. In addition, 12 patients received second-line TMZ therapy as they had received 6 courses of adjuvant TMZ therapy and did not have disease recurrence for $>6$ months: due to the limited number of patients receiving this therapeutic option, this treatment was excluded when analyzing the effect of the different second-line treatments on survival. Compared to patients who received no second-line therapy, there was a significant OS increase in those who underwent reoperation (hazard ratio $(\mathrm{HR})=0.39 ; 95 \% \mathrm{CI}, 0.25-0.60$ ) or received BEV/IRI therapy ( $\mathrm{HR}=0.23$; $95 \% \mathrm{CI}, 0.15-0.34$ ) as single treatments. When comparing OS for patients who received $\mathrm{BEV} / \mathrm{IRI}$ as single second-line therapy with those who received a combination of reoperation plus second-line BEV/IRI therapy, there was no significant beneficial effect, although there was a tendency for better survival among those who received the combination $(\mathrm{HR}=0.87 ; 95 \% \mathrm{CI}$, $0.46-1.37)$. In contrast, when the reoperation-BEV/IRI combination was compared to reoperation alone, there was a significant increase in survival $(\mathrm{HR}=0.51 ; 95 \%$ CI, 0.31-0.83).

\section{A prognostic model can predict survival of GBM patients receiving $R T / T M Z$}

Multivariable analyses of TTP and OS were done including the covariates described in Tables 2 and 3.

Multivariable analysis of the secondary endpoint, TTP, yielded a final model only including corticosteroid therapy (yes vs no, $\mathrm{HR}=1.41$ (95\% CI, 1.02-1.92), $\mathrm{p}=0.036$ ). The p-values to include ECOG PS and age in the final model were 0.12 and 0.21 respectively. The p-values to include the remaining covariates were all $>0.14$.

A final model was selected for the primary endpoint OS, the following covariates were statistically significant ECOG PS (PS 1 vs $0, \mathrm{HR}=1.22$ (95\% CI, 0.89-1.68), PS 2 vs $0, \mathrm{HR}=2.06(95 \% \mathrm{CI}, 1.25-3.42), \mathrm{p}=0.015)$, corticosteroid therapy (yes vs no, $\mathrm{HR}=2.06(95 \% \mathrm{CI}$, $1.47-2.87$ ), $\mathrm{p}<0.0001$ ) and age (per 10 years, $\mathrm{HR}=1.31$ (95\% CI, 1.11-1.54), $\mathrm{p}=0.001$ ). $\mathrm{P}$ values to include the excluded covariates in the final model were $>0.17$ (P53, $\mathrm{p}=0.57$; MGMT, $\mathrm{p}=0.24$; EGFR, $\mathrm{p}=0.45$; tumor size, $\mathrm{p}=0.51$; operation, $\mathrm{p}=0.84$; multifocal $v$ s. single lesion, $\mathrm{p}=0.17$ ). Significant interactions could not be demonstrated suggesting an additive effect of these covariates. Model assessment was found to adequate. The overall concordance index (C-index) [35-37] for the final model was 0.82 (95\% CI, 0.71-0.92), which can be interpreted as the probability of concordance between predicted and observed survival, thereby demonstrating a substantial discrimination for this model. The results of the five-fold internal cross validation supported the chosen model validating the model in the 5 test sets $(\mathrm{C}$-indices $>0.80)$.

Based on estimated regression coefficients, patient survival chances at $6,12,18$, and 24 months after diagnosis were calculated for various levels of each of the three covariates (Table 4). For example, the survival probability for a 40-year-old patient with ECOG PS 0 receiving no corticosteroid therapy was $97 \%, 86 \%, 73 \%$, and $64 \%$ at 6 , 12,18 and 24 months, respectively, following diagnosis. A much lower survival probability is exemplified for a 80-year-old patient with ECOG PS 2 receiving corticosteroids: $67 \%, 15 \%, 2 \%$, and $0 \%$ at $6,12,18$, and 24 months, respectively from diagnosis. It can also be seen that a change in several variables at the same time can have a major negative impact on the survival probability for the individual patient, while a change in only one of the three factors had a relatively minor impact on the survival probability. This is exemplified by a survival probability of $24 \%$ at 12 months after diagnosis for a 70 -year-old patient with ECOG PS 2 receiving corticosteroid therapy compared to $67 \%$ for a 50 -year-old patient with ECOG PS 0 receiving corticosteroid therapy, and $82 \%$ for a 50 -year-old patient with ECOG PS 0 not receiving corticosteroid therapy. It is noteworthy that a 20 -year increase of patient age has a negative effect on survival probability that is similar to that seen for an increase in ECOG PS from 0 to 2 or corticosteroid therapy $v s$. no therapy.

\section{Discussion}

In this study, we examined a cohort of newly diagnosed GBM patients treated with RT plus concomitant and adjuvant TMZ as primary therapy. We observed a median OS of 14.3 months and a median TTP of 8.0 months (Figure 2), which is very similar to values found in the EORTC-NCIC trial (14.6 and 6.9 months respectively) [2]. Based on this and the fact that the examined patients were consecutive and not selected, we conclude that the patients included are good representatives for the general population affected with GBM.

As treatment for recurrent disease, we found that both BEV/IRI therapy and reoperation resulted in significantly increased OS compared to untreated patients, which is in line with other studies [25,38]. In addition, our results indicate that BEV/IRI therapy is more effective than reoperation as second-line therapy for the majority of patients with recurrent GBM tumors and that the therapy should be given in combination with reoperation when possible. However, as the second-line treatments were based on individual evaluation of patient health status and not on a randomized trial, this could result from the fact that mainly the best performing patients received the reoperation and BEV/IRI combination. Randomized clinical 
Table 4 Estimated survival probabilities from diagnosis depending on patient ECOG PS, corticosteroid therapy use and age

\begin{tabular}{|c|c|c|c|c|c|c|}
\hline \multirow[t]{2}{*}{ ECOG PS } & \multirow{2}{*}{$\begin{array}{l}\text { Steroid } \\
\text { therapy }\end{array}$} & \multirow{2}{*}{$\begin{array}{c}\text { Age } \\
\text { (years) }\end{array}$} & \multicolumn{4}{|c|}{ Survival probability (\%) } \\
\hline & & & $\begin{array}{c}\text { At } 6 \\
\text { months }\end{array}$ & $\begin{array}{c}\text { At } 12 \\
\text { months }\end{array}$ & $\begin{array}{c}\text { At } 18 \\
\text { months }\end{array}$ & $\begin{array}{c}\text { At } 24 \\
\text { months } \\
\end{array}$ \\
\hline \multirow[t]{18}{*}{0} & No & 40 & 97 & 86 & 73 & 64 \\
\hline & & 45 & 96 & 84 & 70 & 60 \\
\hline & & 50 & 96 & 82 & 66 & 56 \\
\hline & & 55 & 95 & 80 & 62 & 51 \\
\hline & & 60 & 95 & 77 & 58 & 46 \\
\hline & & 65 & 94 & 74 & 54 & 41 \\
\hline & & 70 & 93 & 71 & 49 & 36 \\
\hline & & 75 & 92 & 68 & 44 & 32 \\
\hline & & 80 & 91 & 64 & 39 & 27 \\
\hline & Yes & 40 & 94 & 73 & 52 & 40 \\
\hline & & 45 & 93 & 70 & 48 & 35 \\
\hline & & 50 & 92 & 67 & 43 & 30 \\
\hline & & 55 & 91 & 63 & 38 & 25 \\
\hline & & 60 & 89 & 59 & 33 & 21 \\
\hline & & 65 & 88 & 54 & 28 & 16 \\
\hline & & 70 & 86 & 50 & 23 & 13 \\
\hline & & 75 & 84 & 45 & 19 & 9 \\
\hline & & 80 & 82 & 40 & 15 & 7 \\
\hline \multirow[t]{18}{*}{1} & No & 40 & 96 & 83 & 68 & 58 \\
\hline & & 45 & 96 & 81 & 64 & 53 \\
\hline & & 50 & 95 & 79 & 60 & 49 \\
\hline & & 55 & 94 & 76 & 56 & 44 \\
\hline & & 60 & 93 & 73 & 52 & 39 \\
\hline & & 65 & 93 & 70 & 47 & 34 \\
\hline & & 70 & 91 & 66 & 42 & 29 \\
\hline & & 75 & 90 & 62 & 37 & 24 \\
\hline & & 80 & 89 & 58 & 32 & 20 \\
\hline & Yes & 40 & 92 & 68 & 45 & 32 \\
\hline & & 45 & 91 & 65 & 40 & 28 \\
\hline & & 50 & 90 & 61 & 35 & 23 \\
\hline & & 55 & 89 & 57 & 30 & 18 \\
\hline & & 60 & 87 & 52 & 26 & 14 \\
\hline & & 65 & 85 & 47 & 21 & 11 \\
\hline & & 70 & 83 & 43 & 17 & 8 \\
\hline & & 75 & 81 & 38 & 13 & 5 \\
\hline & & 80 & 79 & 33 & 10 & 4 \\
\hline \multirow[t]{5}{*}{2} & No & 40 & 94 & 73 & 52 & 40 \\
\hline & & 45 & 93 & 70 & 47 & 35 \\
\hline & & 50 & 92 & 67 & 43 & 30 \\
\hline & & 55 & 90 & 63 & 38 & 25 \\
\hline & & 60 & 89 & 59 & 33 & 20 \\
\hline
\end{tabular}

Table 4 Estimated survival probabilities from diagnosis depending on patient ECOG PS, corticosteroid therapy use and age (Continued)

\begin{tabular}{cccccc}
\hline 65 & 88 & 54 & 28 & 16 \\
& 70 & 86 & 50 & 23 & 12 \\
& 75 & 84 & 45 & 19 & 9 \\
& 80 & 82 & 40 & 15 & 7 \\
\hline Yes & 40 & 87 & 53 & 26 & 15 \\
& 45 & 85 & 48 & 22 & 11 \\
& 50 & 84 & 43 & 17 & 8 \\
55 & 81 & 38 & 13 & 6 \\
60 & 79 & 33 & 10 & 4 \\
65 & 76 & 28 & 7 & 2 \\
& 70 & 73 & 24 & 5 & 1 \\
75 & 70 & 19 & 3 & 1 \\
& 80 & 67 & 15 & 2 & 0 \\
\hline
\end{tabular}

trials are therefore needed for a better comparison of these two different second-line treatments.

Although RT/TMZ improves survival as compared to patients receiving $\mathrm{RT}$ alone, it only results in long-term survival ( $>2$ years) for $<30 \%$ of patients [10]. Much effort has been devoted to finding parameters that correlate with response to and survival following RT/TMZ therapy. Using univariate analysis in the present study, we found that three clinical markers (age, ECOG PS, and corticosteroid therapy at treatment initiation) had a significant impact on survival following therapy (Table 2). All three variables have been previously reported to affect survival. However, while an analysis of the EORTC-NCIC trial data was able to find an impact of all three variables [14], studies on other patient groups only saw a significant effect for one of these markers $[12,16]$.

Contrary to our expatiations, we were not able to find any significance from the extent of primary operation in our study (Table 2), although several other studies have shown a significant effect for this variable on the response and survival of GBM patients treated with RT/TMZ [13-15,39]. As in other studies with similar negative results $[12,40]$, we expect that the non-significant result is caused by incorrect assessment of surgical radicality, which in this study was estimated based on surgeons' impression of tumor remaining in the resection area. Supporting this is the significant effect observed in our study for second-line reoperation, which was performed by a more experienced team of surgeons at our institution. Our results underline the importance of standardizing the evaluation process, in which the use of early (within 72 hours of surgery) MRI scans could be an important tool, a method used in several studies finding an effect of primary surgery $[13,39]$. 
There is a strong indication for the involvement of EGFR and p53 in the response of GBM to TMZ. Studies on GBM cells couple signaling from the EGFR receptor to reduced sensitivity to chemotherapeutic agents that, like TMZ, have alkylating activity [41,42], while p53 inactivation in GBM cells results in increased TMZ sensitivity $[43,44]$. However, in line with previous studies examining the prognostic value of EGFR [13,18,40,45] in TMZ-treated GBM patients, we were unable to find a significant correlation between this molecule and patient response or survival (Table 3 ). We found a significantly increased response rate in patients who had p53-positive tumors compared to those with p53-negative tumors, although we were unable to find a significant effect on OS and TTP. This adds to the conflicting picture existing for this molecule, for which both significant and non-significant results exist regarding its effect on response and survival $[13,18,40,45]$. Overall, these results indicate that EGFR and p53, despite their involvement in GBM tumor development and growth, not are main players in the response of GBM tumors to TMZ. However, improved assay techniques and consideration of tumor heterogeneity are necessary to confirm this.

Many studies have shown a significant correlation between lack of MGMT expression and survival of TMZ-treated GBM patients $[11,13,14]$. However, the detection method varies from direct detection of the MGMT protein to indirect detection of the methylation status of the MGMT promoter as a marker for its expression [46]. In line with previous studies [12,13], we were unable to show a significant correlation between MGMT status and outcome following RT/TMZ therapy when detecting MGMT at the protein level using IHC. This, combined with an analysis which found that MGMT protein expression does not correlate with the promoter methylation status of MGMT [47], indicates that IHC is not a reliable technique for MGMT detection for prediction of patient response to TMZ.

Emerging results show that GBM tumors can be subclassified into different groups based on their molecular expression patterns and that these subclasses correlate to variations in patient survival $[48,49]$. This observation indicates that individualized therapy could be a way to increase the survival of GBM patients.

Research conducted on parameters that are able to predict response and survival following TMZ therapy has mostly centered on single markers. This has resulted in the identification of a number of both clinical and molecular parameters [11-13,16], but none of these have been able to give an accurate prediction of RT/TMZ therapy outcome for the individual patient. As a result, no markers have been implemented to segregate patients into responders and non-responders for RT/TMZ therapy, although the combination has been given as standard therapy for GBM patients since 2005. That an approach taking several markers into account simultaneously is beneficial is indicated by the ability of the RPA classification system to subgroup RT/TMZ-treated patients according to survival [27] and by studies that are able to increase the predictive effect using multigene [50] or multimethylation [51] profiles as compared to the use of single variables.

Based on these facts, we assembled a model to predict patient survival using the individual variables that we had identified as significant for survival (age, ECOG PS and corticosteroid therapy at treatment initiation). The model, which was developed using cox modelling, is able to calculate the probability for a given patient receiving the described therapy to be alive at a given time and can be used to identify patients with the best and worst survival chances (Table 4). Another approach could be recursive partitioning, thereby making a decision tree model as used in the RPA classification system. However, as discussed previously $[14,28]$, this approach groups the variables into only a few categories and cannot predict the survival for the individual patient.

Furthermore, our model contributes to the debate on which therapeutic option should be preferred for elderly patients [52]. Both RT [53] and TMZ [54] have been proven to result in survival benefit for elderly GBM patients. However, due to the general belief that elderly patients do not tolerate concomitant chemoradiotherapy as well as younger patients in combination with the observation of a negative correlation between patient age and the survival following RT/TMZ therapy [14], this combination is not standard in elderly patients. Our results indicate that age alone should not disqualify patients from concomitant RT/TMZ therapy, but that ECOG PS and use of corticosteroid therapy should be taken into account for making any therapeutic decisions. This conclusion supports several studies which have found that RT/TMZ therapy is effective in elderly GBM patients presenting with good prognostic factors [15,55].

A few other studies have constructed prognostic models for GBM patients. One model established from patients receiving $\mathrm{RT} / \mathrm{TMZ}$ as primary treatment in the EORTCNCIC trials includes age, PS, MGMT status, extent of resection, and mental state [14]. Another model based on GBM patients receiving RT/TMZ therapy for recurrent disease includes PS, corticosteroid therapy, number of lesions, and lesion size [29]. As PS is the only consistent factor in all three studies, additional research is needed. Nonetheless, the described prognostic models have the potential to be valuable tools for clinicians when deciding which therapeutic modality is the best for the individual GBM patients. 


\section{Conclusions}

This study demonstrates a significant impact of patient age, ECOG PS and status of corticosteroid therapy on TTP and OS for GBM patients treated with RT/TMZ as primary therapy and re-operation or BEV/IRI as secondary therapy. Further by assembling these variables in a model the survival chances at different time-points from diagnosis can be predicted for GBM patients receiving the described therapy.

\section{Competing interests}

The authors declare that they have no competing interests.

\section{Author's contributions}

SRM and HSP have contributed with the design of the study, interpretation of data and drafting of manuscript. IJC has contributed substantially to the analysis and interpretation of data. KG and HB have contributed by the collection and analysis of data. M-TS and MK have contributed substantially to the manuscript drafting and revision. All authors read and approved the final manuscript

\section{Acknowledgments}

Financial support was kindly provided by Aase and Ejnar Danielsens Foundation, Kathrine and Vigo Skovgaards Foundation, and the I.M. Daehnfeldt Foundation. Editorial support was provided by Miller Medical Communications (Brindle, Lancashire, UK).

\section{Author details}

'Department of Radiation Biology, The Finsen Center, Copenhagen University Hospital, Blegdamsvej 9, DK-2100 Copenhagen, Denmark. ${ }^{2}$ The Finsen Laboratory, Copenhagen University Hospital, Blegdamsvej 9, DK-2100 Copenhagen and Biotech Research and Innovation Center (BRIC), University of Copenhagen, Ole Maaløes Vej 5, DK-2200 Copenhagen, Denmark ${ }^{3}$ Department of Neuropathology, Center of Diagnostic Investigation, Copenhagen University Hospital, Blegdamsvej 9, DK-2100 Copenhagen, Denmark. ${ }^{4}$ Department of Neurosurgery, The Neurocenter, Copenhagen University Hospital, Blegdamsvej 9, DK-2100 Copenhagen, Denmark.

\section{Received: 7 February 2013 Accepted: 28 August 2013}

Published: 3 September 2013

\section{References}

1. Ohgaki $\mathrm{H}$, Kleihues P: Epidemiology and etiology of gliomas. Acta Neuropathol 2005, 109:93-108.

2. Stupp R, Mason WP, van den Bent MJ, Weller M, Fisher B, Taphoorn MJ, Belanger K, Brandes AA, Marosi C, Bogdahn U, Curschmann J, Janzer RC, Ludwin SK, Gorlia T, Allgeier A, Lacombe D, Cairncross JG, Eisenhauer E, Mirimanoff RO: Radiotherapy plus concomitant and adjuvant temozolomide for glioblastoma. N Engl J Med 2005, 352:987-996.

3. Ryken TC, Frankel B, Julien T, Olson JJ: Surgical management of newly diagnosed glioblastoma in adults: role of cytoreductive surgery. J Neurooncol 2008, 89:271-286.

4. Sanai N, Berger MS: Recent surgical management of gliomas. Adv Exp Med Biol 2012, 746:12-25.

5. Quick A, Patel D, Hadziahmetovic M, Chakravarti A, Mehta M: Current therapeutic paradigms in glioblastoma. Rev Recent Clin Trials 2010, 5:14-27.

6. Fukushima $\mathrm{T}$, Takeshima $\mathrm{H}$, Kataoka $\mathrm{H}$ : Anti-glioma therapy with temozolomide and status of the DNA-repair gene MGMT. Anticancer Res 2009, 29:4845-4854.

7. Yung WK, Albright RE, Olson J, Fredericks R, Fink K, Prados MD, Brada M, Spence A, Hohl RJ, Shapiro W, Glantz M, Greenberg H, Selker RG, Vick NA, Rampling R, Friedman H, Phillips P, Bruner J, Yue N, Osoba D, Zaknoen S, Levin VA: A phase II study of temozolomide vs. procarbazine in patients with glioblastoma multiforme at first relapse. $\mathrm{Br} J$ Cancer 2000, 83:588-593.

8. Wedge SR, Porteous JK, Glaser MG, Marcus K, Newlands ES: In vitro evaluation of temozolomide combined with X-irradiation. Anticancer Drugs 1997, 8:92-97.
9. Chakravarti A, Erkkinen MG, Nestler U, Stupp R, Mehta M, Aldape K, Gilbert MR, Black PM, Loeffler JS: Temozolomide-mediated radiation enhancement in glioblastoma: a report on underlying mechanisms. Clin Cancer Res 2006, 12:4738-4746.

10. Stupp R, Hegi ME, Mason WP, van den Bent MJ, Taphoorn MJ, Janzer RC, Ludwin SK, Allgeier A, Fisher B, Belanger K, Hau P, Brandes AA, Gijtenbeek J, Marosi C, Vecht CJ, Mokhtari K, Wesseling P, Villa S, Eisenhauer E, Gorlia T, Weller M, Lacombe D, Cairncross JG, Mirimanoff RO: Effects of radiotherapy with concomitant and adjuvant temozolomide versus radiotherapy alone on survival in glioblastoma in a randomised phase III study: 5-year analysis of the EORTC-NCIC trial. Lancet Oncol 2009, 10:459-466.

11. Hegi ME, Diserens AC, Gorlia T, Hamou MF, De TN, Weller M, Kros JM, Hainfellner JA, Mason W, Mariani L, Bromberg JE, Hau P, Mirimanoff RO, Cairncross JG, Janzer RC, Stupp R: MGMT gene silencing and benefit from temozolomide in glioblastoma. N Engl J Med 2005, 352:997-1003.

12. Iliadis G, Kotoula V, Chatzisotiriou A, Televantou D, Eleftheraki AG, Lambaki S, Misailidou D, Selviaridis P, Fountzilas G: Volumetric and MGMT parameters in glioblastoma patients: Survival analysis. BMC Cancer 2012, 12:3.

13. Felsberg J, Rapp M, Loeser S, Fimmers R, Stummer W, Goeppert M, Steiger $H J$, Friedensdorf B, Reifenberger G, Sabel MC: Prognostic significance of molecular markers and extent of resection in primary glioblastoma patients. Clin Cancer Res 2009, 15:6683-6693.

14. Gorlia T, van den Bent MJ, Hegi ME, Mirimanoff RO, Weller M, Cairncross JG, Eisenhauer E, Belanger K, Brandes AA, Allgeier A, Lacombe D, Stupp R: Nomograms for predicting survival of patients with newly diagnosed glioblastoma: prognostic factor analysis of EORTC and NCIC trial 26981-22981/CE.3. Lancet Oncol 2008, 9:29-38.

15. Combs SE, Wagner J, Bischof M, Welzel T, Wagner F, Debus J, Schulz-Ertner $D$ : Postoperative treatment of primary glioblastoma multiforme with radiation and concomitant temozolomide in elderly patients. Int J Radiat Oncol Biol Phys 2008, 70:987-992.

16. Jeon HJ, Kong DS, Park KB, Lee JI, Park K, Kim JH, Kim ST, Lim dH, Kim WS, Nam DH: Clinical outcome of concomitant chemoradiotherapy followed by adjuvant temozolomide therapy for glioblastaomas: single-center experience. Clin Neurol Neurosurg 2009, 111:679-682.

17. Verbeek B, Southgate TD, Gilham DE, Margison GP: O6-Methylguanine-DNA methyltransferase inactivation and chemotherapy. Br Med Bull 2008, 85:17-33.

18. Malkoun N, Chargari C, Forest F, Fotso MJ, Cartier L, Auberdiac P, Thorin J, Pacaut C, Peoc'h M, Nuti C, Schmitt T, Magne N: Prolonged temozolomide for treatment of glioblastoma: preliminary clinical results and prognostic value of p53 overexpression. J Neurooncol 2012, 106:127-133.

19. Hobbs J, Nikiforova MN, Fardo DW, Bortoluzzi S, Cieply K, Hamilton RL, Horbinski C: Paradoxical relationship between the degree of EGFR amplification and outcome in glioblastomas. Am J Surg Pathol 2012, 36:1186-1193.

20. Hochberg $\mathrm{FH}$, Pruitt $\mathrm{A}$ : Assumptions in the radiotherapy of glioblastoma. Neurology 1980, 30:907-911.

21. Park JK, Hodges T, Arko L, Shen M, Dello ID, McNabb A, Olsen BN, Kreis IN, Iwamoto FM, Sul J, Auh S, Park GE, Fine HA, Black PM: Scale to predict survival after surgery for recurrent glioblastoma multiforme. J Clin Oncol 2010, 28:3838-3843.

22. Reardon DA, Turner S, Peters KB, Desjardins A, Gururangan S, Sampson JH, McLendon RE, Herndon JE, Jones LW, Kirkpatrick JP, Friedman AH, Vredenburgh JJ, Bigner DD, Friedman HS: A review of VEGF/VEGFRtargeted therapeutics for recurrent glioblastoma. J Natl Compr Canc Netw 2011, 9:414-427

23. Brem S, Cotran R, Folkman J: Tumor angiogenesis: a quantitative method for histologic grading. J Natl Cancer Inst 1972, 48:347-356.

24. Salmaggi A, Eoli M, Frigerio S, Silvani A, Gelati M, Corsini E, Broggi G, Boiardi A: Intracavitary VEGF, bFGF, IL-8, IL-12 levels in primary and recurrent malignant glioma. J Neurooncol 2003, 62:297-303.

25. Chamberlain MC: Bevacizumab for the treatment of recurrent glioblastoma. Clin Med Insights Oncol 2011, 5:117-129.

26. Curran WJ Jr, Scott CB, Horton J, Nelson JS, Weinstein AS, Fischbach AJ, Chang $\mathrm{CH}$, Rotman M, Asbell SO, Krisch RE: Recursive partitioning analysis of prognostic factors in three Radiation Therapy Oncology Group malignant glioma trials. J Natl Cancer Inst 1993, 85:704-710. 
27. Mirimanoff RO, Gorlia T, Mason W, van den Bent MJ, Kortmann RD, Fisher B, Reni M, Brandes AA, Curschmann J, Villa S, Cairncross G, Allgeier A, Lacombe D, Stupp R: Radiotherapy and temozolomide for newly diagnosed glioblastoma: recursive partitioning analysis of the EORTC 26981/22981-NCIC CE3 phase III randomized trial. J Clin Oncol 2006, 24:2563-2569

28. Li J, Wang M, Won M, Shaw EG, Coughlin C, Curran WJ Jr, Mehta MP: Validation and simplification of the Radiation Therapy Oncology Group recursive partitioning analysis classification for glioblastoma. Int J Radiat Oncol Biol Phys 2011, 81:623-630.

29. Gorlia T, Stupp R, Brandes AA, Rampling RR, Fumoleau P, Dittrich C, Campone MM, Twelves CC, Raymond E, Hegi ME, Lacombe D, van den Bent MJ: New prognostic factors and calculators for outcome prediction in patients with recurrent glioblastoma: a pooled analysis of EORTC Brain Tumour Group phase I and II clinical trials. Eur J Cancer 2012, 48:1176-1184.

30. Emami B, Lyman J, Brown A, Coia L, Goitein M, Munzenrider JE, Shank B, Solin LJ, Wesson M: Tolerance of normal tissue to therapeutic irradiation. Int J Radiat Oncol Biol Phys 1991, 21:109-122.

31. Poulsen HS, Grunnet K, Sorensen M, Olsen P, Hasselbalch B, Nelausen K, Kosteljanetz M, Lassen U: Bevacizumab plus irinotecan in the treatment patients with progressive recurrent malignant brain tumours. Acta Oncol 2009, 48:52-58.

32. Oken MM, Creech $\mathrm{RH}$, Tormey DC, Horton J, Davis TE, McFadden ET, Carbone PP: Toxicity and response criteria of the Eastern Cooperative Oncology Group. Am J Clin Oncol 1982, 5:649-655.

33. $\mathrm{NCl}$ common terminology criteria for adverse events v.3.0 (CTCAE). [http://ctep. cancer.gov/forms/docs/ctcae_laypublish.xls] Accessed January 92013.

34. Macdonald DR, Cascino TL, Schold SC Jr, Cairncross JG: Response criteria for phase II studies of supratentorial malignant glioma. J Clin Oncol 1990, 8:1277-1280.

35. Harrell FE Jr, Lee KL, Mark DB: Multivariable prognostic models: issues in developing models, evaluating assumptions and adequacy, and measuring and reducing errors. Stat Med 1996, 15:361-387.

36. Pencina MJ, D'Agostino RB: Overall $C$ as a measure of discrimination in survival analysis: model specific population value and confidence interval estimation. Stat Med 2004, 23:2109-2123.

37. Liu L, Forman S, Barton B: Fitting Cox model using PROC PHREG and beyond in SAS. In SAS Institute Inc. 2009. Proceedings of the SAS ${ }^{\circledR} \mathrm{Global}$ Forum 2009 Conference, Paper 236-2009. Cary, NC: SAS Institute Inc::1-10.

38. Barbagallo GM, Jenkinson MD, Brodbelt AR: 'Recurrent' glioblastoma multiforme, when should we reoperate? Br J Neurosurg 2008, 22:452-455.

39. Li S, Zhang W, Chen B, Jiang T, Wang Z: Prognostic and predictive value of p53 in low MGMT expressing glioblastoma treated with surgery, radiation and adjuvant temozolomide chemotherapy. Neurol Res 2010 32:690-694.

40. Ang C, Guiot MC, Ramanakumar AV, Roberge D, Kavan P: Clinical significance of molecular biomarkers in glioblastoma. Can J Neurol Sci 2010, 37:625-630.

41. Chakravarti A, Chakladar A, Delaney MA, Latham DE, Loeffler JS: The epidermal growth factor receptor pathway mediates resistance to sequential administration of radiation and chemotherapy in primary human glioblastoma cells in a RAS-dependent manner. Cancer Res 2002, 62:4307-4315.

42. Nagane M, Narita Y, Mishima K, Levitzki A, Burgess AW, Cavenee WK, Huang $\mathrm{HJ}$ : Human glioblastoma xenografts overexpressing a tumor-specific mutant epidermal growth factor receptor sensitized to cisplatin by the AG1478 tyrosine kinase inhibitor. J Neurosurg 2001, 95:472-479.

43. Blough MD, Beauchamp DC, Westgate MR, Kelly JJ, Cairncross JG: Effect of aberrant p53 function on temozolomide sensitivity of glioma cell lines and brain tumor initiating cells from glioblastoma. J Neurooncol 2011, 102:1-7.

44. Dinca EB, Lu KV, Sarkaria JN, Pieper RO, Prados MD, Haas-Kogan DA, Vandenberg SR, Berger MS, James CD: p53 Small-molecule inhibitor enhances temozolomide cytotoxic activity against intracranial glioblastoma xenografts. Cancer Res 2008, 68:10034-10039.

45. Brown PD, Krishnan S, Sarkaria JN, Wu W, Jaeckle KA, Uhm JH, Geoffroy FJ, Arusell R, Kitange G, Jenkins RB, Kugler JW, Morton RF, Rowland KM Jr, Mischel P, Yong WH, Scheithauer BW, Schiff D, Giannini C, Buckner JC: Phase I/II trial of erlotinib and temozolomide with radiation therapy in the treatment of newly diagnosed glioblastoma multiforme: North Central Cancer Treatment Group Study N0177. J Clin Oncol 2008, 26:5603-5609.
46. Quillien V, Lavenu A, Karayan-Tapon L, Carpentier C, Labussiere M, Lesimple T, Chinot O, Wager M, Honnorat J, Saikali S, Fina F, Sanson M, FigarellaBranger D: Comparative assessment of 5 methods (methylation-specific polymerase chain reaction, MethyLight, pyrosequencing, methylationsensitive high-resolution melting, and immunohistochemistry) to analyze O6-methylguanine-DNA-methyltranferase in a series of 100 glioblastoma patients. Cancer 2012, 118:4201-4211.

47. Brell M, Ibanez J, Tortosa A: O6-Methylguanine-DNA methyltransferase protein expression by immunohistochemistry in brain and non-brain systemic tumours: systematic review and meta-analysis of correlation with methylation-specific polymerase chain reaction. BMC Cancer 2011, 11:35.

48. Phillips HS, Kharbanda S, Chen R, Forrest WF, Soriano RH, Wu TD, Misra A, Nigro JM, Colman H, Soroceanu L, Williams PM, Modrusan Z, Feuerstein BG, Aldape K: Molecular subclasses of high-grade glioma predict prognosis, delineate a pattern of disease progression, and resemble stages in neurogenesis. Cancer Cell 2006, 9:157-173.

49. Verhaak RG, Hoadley KA, Purdom E, Wang V, Qi Y, Wilkerson MD, Miller CR, Ding L, Golub T, Mesirov JP, Alexe G, Lawrence M, O'Kelly M, Tamayo P, Weir BA, Gabriel S, Winckler W, Gupta S, Jakkula L, Feiler HS, Hodgson JG, James CD, Sarkaria JN, Brennan C, Kahn A, Spellman PT, Wilson RK, Speed TP, Gray JW, et al: Integrated genomic analysis identifies clinically relevant subtypes of glioblastoma characterized by abnormalities in PDGFRA, IDH1, EGFR, and NF1. Cancer Cell 2010, 17:98-110.

50. Colman H, Zhang L, Sulman EP, McDonald JM, Shooshtari NL, Rivera A, Popoff S, Nutt CL, Louis DN, Cairncross JG, Gilbert MR, Phillips HS, Mehta MP, Chakravarti A, Pelloski CE, Bhat K, Feuerstein BG, Jenkins RB, Aldape K: A multigene predictor of outcome in glioblastoma. Neuro Oncol 2010 12:49-57.

51. van den Bent MJ, Gravendeel LA, Gorlia T, Kros JM, Lapre L, Wesseling P, Teepen $J$, Idbaih A, Sanson M, Smitt PA, French PJ: A hypermethylated phenotype is a better predictor of survival than MGMT methylation in anaplastic oligodendroglial brain tumors: a report from EORTC study 26951. Clin Cancer Res 2011, 17:7148-7155.

52. Brandes AA, Bartolotti M: Neuro-oncology: treatment decisions in elderly patients with glioblastoma. Nat Rev Neurol 2012, 8:664-665.

53. Keime-Guibert F, Chinot O, Taillandier L, Cartalat-Carel S, Frenay M, Kantor G, Guillamo JS, Jadaud E, Colin P, Bondiau PY, Menei P, Loiseau H, Bernier V, Honnorat J, Barrie M, Mokhtari K, Mazeron JJ, Bissery A, Delattre JY: Radiotherapy for glioblastoma in the elderly. N Engl J Med 2007 356:1527-1535.

54. Malmstrom A, Gronberg BH, Marosi C, Stupp R, Frappaz D, Schultz H, Abacioglu U, Tavelin B, Lhermitte B, Hegi ME, Rosell J, Henriksson R: Temozolomide versus standard 6-week radiotherapy versus hypofractionated radiotherapy in patients older than 60 years with glioblastoma: the Nordic randomised, phase 3 trial. Lancet Oncol 2012, 13:916-926.

55. Minniti G, De SV, Muni R, Filippone F, Bozzao A, Valeriani M, Osti MF, De PU, Lanzetta G, Tombolini V, Maurizi ER: Radiotherapy plus concomitant and adjuvant temozolomide for glioblastoma in elderly patients. J Neurooncol 2008, 88:97-103.

doi:10.1186/1471-2407-13-402

Cite this article as: Michaelsen et al:: Clinical variables serve as prognostic factors in a model for survival from glioblastoma multiforme: an observational study of a cohort of consecutive non-selected patients from a single institution. BMC Cancer 2013 13:402. 